\title{
Tratamiento Temprano de las Maloclusiones sin Aparatología Funcional: Presentación de Dos Casos Clínicos
}

\author{
Early Malocclusion Treatment without Functional Devices: \\ Presentation of Two Clinical Cases
}

\author{
Lorena Carbone Irujo*,**
}

CARBONE, I. L. Tratamiento temprano de las maloclusiones sin aparatología funcional: presentación de dos casos clínicos. Int. J. Odontostomat., 8(2):253-260, 2014.

RESUMEN: En el presente artículo se expone un modelo de trabajo basado en la Rehabilitación Neuro-oclusal (RNO) de Pedro Planas (1997) que "se fundamenta en descubrir dónde, cuándo y cómo hay que actuar sobre los centros neurales receptores que proporcionan la respuesta de desarrollo del sistema estomatognático para que, excitándolos fisiológicamente nos proporcionen una respuesta de desarrollo normal y equilibrado". La RNO busca pasar de un plano oclusal patológico a un plano oclusal funcional, cambiando la postura mandibular, permitiendo un crecimiento y desarrollo eugnácico de la cara.Los casos clínicos relatados en el presente trabajo son relativamente comunes en la práctica clínica odontológica y con su presentación se procura enfatizar la importancia del diagnóstico temprano de la maloclusión y de la intervención terapéutica oportuna; prevenir complicaciones y simplificar el tratamiento, favoreciendo el crecimiento y desarrollo morfofuncional equilibrado del paciente, reflejado directamente y positivamente sobre su estética facial. Se ilustran dos casos clínicos, uno en dentición decidua y el otro en dentición mixta temprana de la clínica de prevención y ortopedia de IUCEDDU; fueron propuestos tratamientos con equilibrado oclusal y de pistas directas Planas, haciendo un seguimiento longitudinal de los casos. Los resultados obtenidos en los tratamientos con este método, reafirman nuestras expectativas y confirman que a pesar del uso de una técnica tan simplificada, se obtienen excelentes resultados que evitan o acortan tratamientos funcionales posteriores.

PALABRAS CLAVE: disgnacia, equilibrado oclusal, pistas directas planas.

\section{INTRODUCCIÓN}

La maloclusión es considerada como el tercer problema odontológico de salud pública. De allí la importancia y responsabilidad por parte de los odontólogos, de diagnosticar e intervenir oportunamente, previniendo la instalación de alteraciones más complejas, dentro del sistema estomatognático en crecimiento y desarrollo (Chibinski et al., 2005).

La etiopatogenia de las maloclusiones en gran parte se explica por las características de la vida de la sociedad contemporánea, y el cambio en los hábitos saludables que eso conlleva. Dentro de la etiopatogenia, cabe resaltar la importancia del amamantamiento por un período adecuado, como estímu- lo fundamental en el desarrollo del sistema estomatognático. Debería extenderse mínimamente durante seis meses e idealmente hasta los dos años del niño, sin embargo ya sea por razones culturales, económicas, debido a la rápida reinserción de la mujer al ámbito laboral no llega a extenderse en esos tiempos estipulados. Esto naturalmente deja secuelas en las estructuras orofaciales del bebé, guiando su crecimiento de manera desequilibrada. Más adelante en el tiempo, es la función masticatoria la que ejerce un rico estímulo semejante, en el crecimiento y desarrollo cráneofacial, pero la alimentación moderna, cada vez más blanda y procesada, no logra la estimulación y correcta maduración del sistema (Planas, 1997).

\footnotetext{
* Universidad de la República, Montevideo, Uruguay.

" Especialista en prevención, ortopedia y ortodoncia Instituto Universitario Centro de Estudio y Diagnóstico de las Disgnacias del Uruguay (IUCEDDU). Ayudante de la clínica de prevención y ortopedia de IUCEDDU a cargo de Prof. Dra. W. Haller y Dra. I.Poggi, IUCEDDU, Montevideo, Uruguay.
} 
El tratamiento temprano implica, el tratamiento de las disgnacias incipientes antes que la cascada etiopatogénica las complejice. No consta solamente de la intervención terapéutica por parte del odontólogo, sino también la orientación previa de las familias, y principalmente de las madres, desde la gestación, sobre los hábitos saludables y sobre la gran importancia del amamantamiento exclusivo. Al mismo tiempo, más adelante, una intervención terapéutica oportuna se hace necesaria y evita el agravamiento de la maloclusión que condenaría al paciente a tratamientos más complejos y largos, con altos índices de recidivas. La edad ideal es la dentición temporaria y mixta temprana donde es posible rehabilitar el sistema masticatorio sin dejar secuelas (Planas; Raymond, 2003; Vesse, 2003; Deshayes, 2006).

Dentro de los recursos terapéuticos indicados en dentición decidua y mixta temprana, están el equilibrado oclusal y las pistas directas (Planas), objeto del presente trabajo en el que se presentarán dos casos de la clínica de ortopedia y prevención del Instituto Universitario Centro de Estudio y Diagnóstico de las Disgnacias del Uruguay (IUCEDDU).

Equilibrado oclusal. Es una técnica que busca modificar la función neuromuscular, a través de la oclusión dentaria; logrando contactos dentarios equilibrados a ambos lados de la línea media y que producirán contracciones musculares armónicas y simétricas de maseteros y temporales.
Se deberá comenzar con un correcto diagnóstico oclusal relajando al niño para realizar el análisis de los ángulos funcionales masticatorios (AFMP) ilustrados en la Figura 1, observando si existen interferencias, que hagan modificar su patrón masticatorio (Planas).

Es importante resaltar que deberá ser realizado por un profesional preparado, con conocimientos para ello, que logre hacer desgastes minuciosos, orientados a eliminar interferencias y contactos prematuros desequilibrantes, que impidan al niño, movimientos mandibulares excursivos amplios. Procurando devolver la funcionalidad adecuada acorde con la edad dentaria del niño.

Esta técnica busca lograr una función masticatoria fisiológica unilateral y alternada. Durante y al finalizar el equilibrado oclusal, se realiza palpación muscular bilateral, en los haces de fibras de los músculos temporales (Fig. 2A) y maseteros (Fig. 2B), de modo de evidenciar clínicamente, la simetría en sus contracciones en oclusión máxima, así como la evaluación del paralelismo entre plano oclusal y plano de Camper, mediante la utilización del plano de Fox (Fig. 2C).

Pistas directas Planas. Constituyen un recurso terapéutico, donde a través de pequeños incrementos de resina compuesta fotopolimerizable, aplicados directamente sobre la superficie oclusal de los dientes caducos, se busca armonizar el plano oclusal en rela-
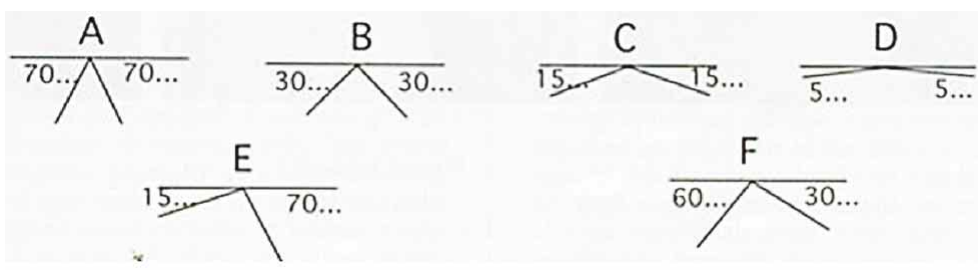

Fig. 1. Ángulos funcionale s masticatorios de Planas (2001).

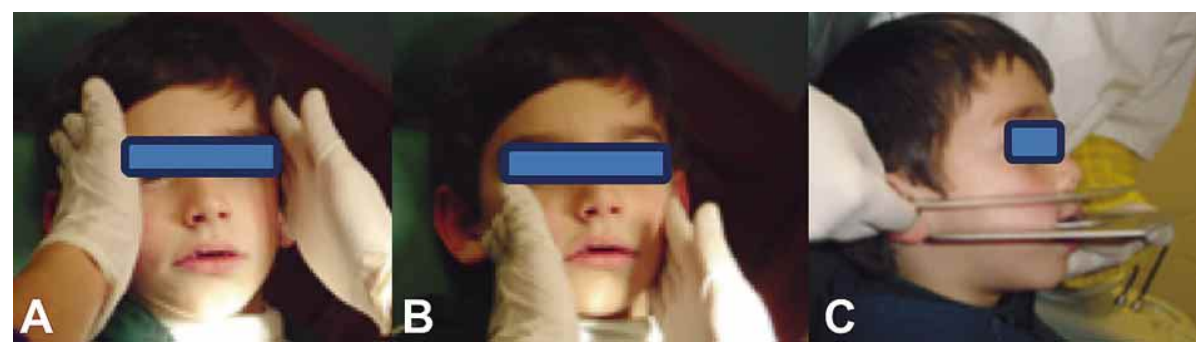

Fig. 2. Palpación muscular bilateral en los músculos temporales $(A)$ y maseteros $(B)$. Evaluación del paralelismo entre plano oclusal y plano de Camper utilizando el plano de Fox (C). 
ción al plano de Camper y eliminar las interferencias para liberar la función mandibular (Planas).

Las pistas ofician de mordida constructiva hendida en una porción maxilar superior y otra maxilar inferior, que tienen por objetivo redirigir el engrama neuromuscular. Posibilitan que el maxilar superior se constituya en una base estable, simetrizada y corregida, en relación al plano de Camper, sobre la cual trabajará la mandíbula remodelándose y relocalizándose.

Ventajas y desventajas: una de las principales ventajas es que permanecen fijas y actúan las 24 horas dentro del sistema estomatognático del niño, garantizando la mantención de una correcta relación intermaxilar durante el desempeño de funciones como la masticación.

Otra ventaja es que no dificultan la dicción del niño, son estéticas y mejoran inmediatamente el desempeño masticatorio del paciente, son de bajo costo y no requieren de un equipamiento ni material especiales y se realizan en pocas sesiones.

Como desventajas se puede citar, que la técnica requiere destreza y conocimiento por parte del profesional y colaboración del niño durante su realización para mantener controlada la humedad, al ser una técnica adhesiva pura.

Mecanismo de acción: generan un cambio en la postura mandibular, modificando la dinámica incorrecta que la disfunción genera en el sistema músculo esquelético; proporcionando de esta forma un desarrollo de la dentición acorde a los patrones normales (Chibinski et al.), a través de la desprogramación de los centros nerviosos adaptados a la disfunción, con posterior remodelación para la posición adecuada.

La nueva postura mandibular determinada por las pistas directas Planas establece una nueva arquitectura para los ciclos masticatorios, produciendo estímulos de crecimiento adecuados y remodelando el sistema estomatognático, así como un nuevo patrón funcional para la lengua, músculos supra e infrahioideos y también masticatorios, faciales y cervicales. A través de una nueva dinámica neuromuscular, se reorienta el crecimiento cráneo facial de acuerdo al potencial individual.

Indicaciones: las pistas directas se indican siempre que se diagnostique un desvío en la trayectoria de cierre, el cual deberá ser corregido siempre y antes de instalar cualquier aparatología de ortopedia. Se utilizan en mayor medida en los casos con diagnóstico de mordida cruzada funcional, mordida cubierta y mordida abierta funcional. En los casos de leve distoclusión permiten el crecimiento mandibular en sentido anteroposterior, al ser liberada la mandíbula; y en prodeslizamientos funcionales, siempre monitoreados mediante controles clínicos periódicos.

Condiciones: estar en período de dentición caduca o mixta temprana, bajo riesgo de caries, tonicidad muscular normal, la presencia de arcadas dentarias armónicas entre sí.

En los casos de disfunción respiratoria, coexistiendo en general con una hipotonía muscular, las expectativas del tratamiento no son las mismas, muchas veces ira acompañado de aparatología funcional.

Técnica: consiste en el agregado de resina compuesta sobre las superficies oclusales de manera de crear superficies masticatorias planas, empezando por el maxilar superior paralelizado al plano de Camper y siguiendo por el inferior. Esto se constata clínicamente mediante la utilización del plano de Fox que nos permite trasladar la posición del plano de Camper sobre el rostro del niño, y así visualizar su relación con el nuevo plano oclusal.

La configuración final de las pistas debe ser una superficie amplia, plataforma oclusal, de modo de mantener una nueva postura terapéutica mandibular, con un espesor suficiente para no fracturarse durante la función oclusal. Deberán confeccionarse manteniendo la relación de las líneas medias dentarias y siempre en armonía con la línea media estética facial.

En muchos casos las pistas Planas reciben una inclinación con finalidad terapéutica, siguiendo los conceptos de Barbosa Dos Santos (2005), recreando un plano oclusal que favorezca los movimientos de lateralidad mandibular.

El monitoreo periódico es imprescindible, observando el estado de las resinas y la función oclusal en máxima intercuspidación y lateralidad mandibular.

\section{Presentación de casos clínicos}

Paciente de sexo masculino de 7 años con dentición mixta temprana, biprotrusión esqueletal y cuerpo mandibular grande. Asimetría facial con desvío del mentón hacia la derecha. Relación canina de clase I y 
clase I molar, mordida abierta anterior, líneas medias no coincidentes. Disfunción respiratoria, masticatoria y deglutoria, láterodesviación funcional y prodeslizamiento al cierre.

Plan de tratamiento: derivación a fonoaudilólogo para reeducación respiratoria y deglutoria; primera fase de equlibrado oclusal para corregir laterodesviación y prodeslizamiento funcional, y mordida abierta anterior. Se plantea evaluar segunda fase con ortopedia funcional.
Fotos de rostro, de frente y perfil al inicio del tratamiento (Fig. 3A-C). Se observa ligera asimetría facial, con desvío del mentón hacia la derecha (Fig. 3B). Fotos intraorales al inicio del tratamiento el año 2008 (Fig. 3D$\mathrm{F})$. Al año se complementa con agregado de resina en piezas 54 y 55 (Fig. 3J-K) para centrar líneas medias dentarias. De acuerdo a la evolución del caso, se decidió no realizar la segunda de aparatología funcional prevista en el plan de tratamiento. En el control el año 2013, despues de 2 años sin maniobras clínicas, se constata la estabilidad del tratamiento (Fig. $4 \mathrm{~A}-\mathrm{C}$ ).

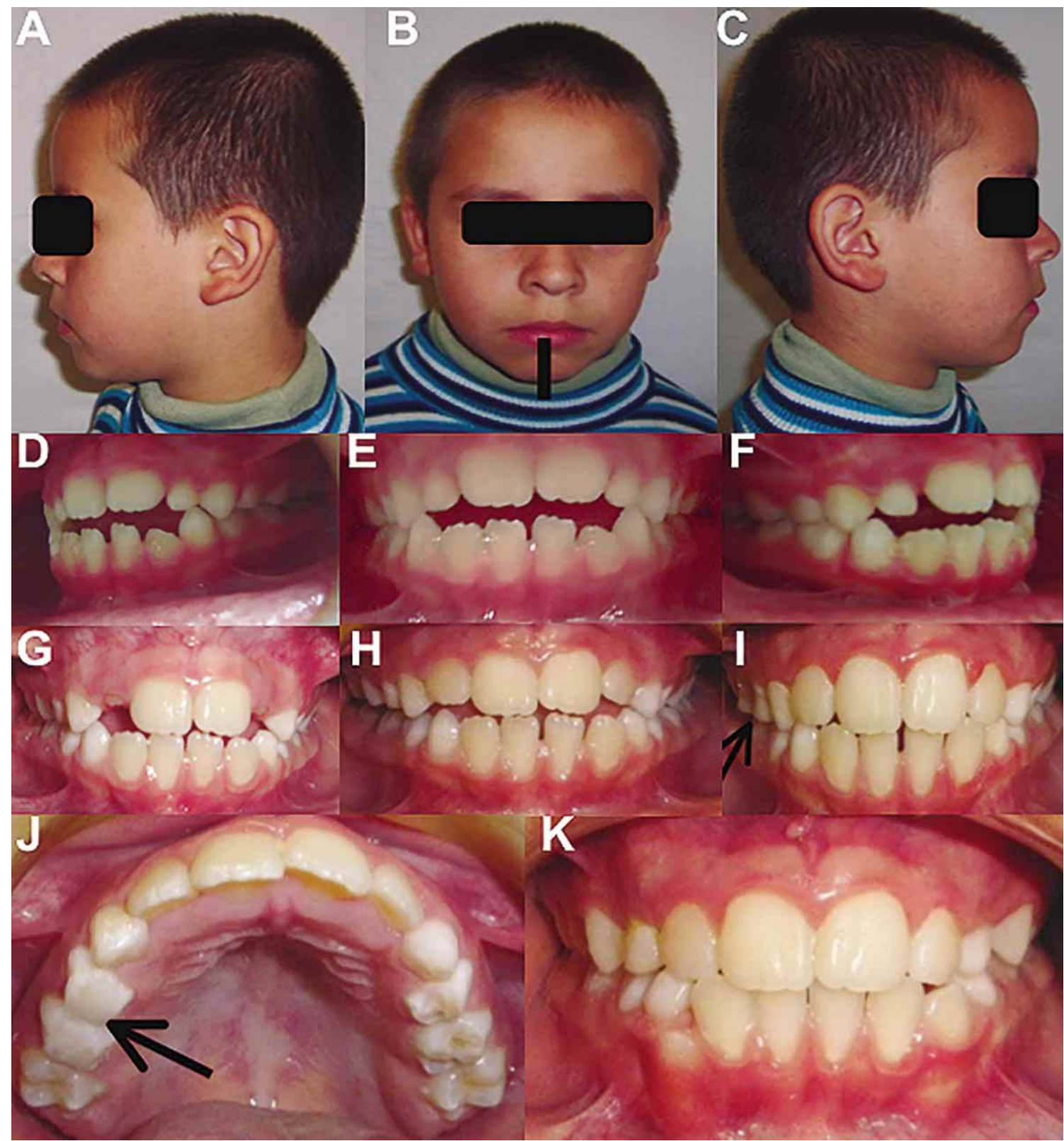

Fig. 3. A. Perfil izquierdo. B. Rostro de frente. C. Perfil derecho. D. Lado izquierdo inicial. E. Oclusión inicial. F. Lado derecho inicial. G. Equilibrado oclusal. H. Control a los 8 meses. I. Control al año. J. Agregado de resina y K. Control año 2011. 


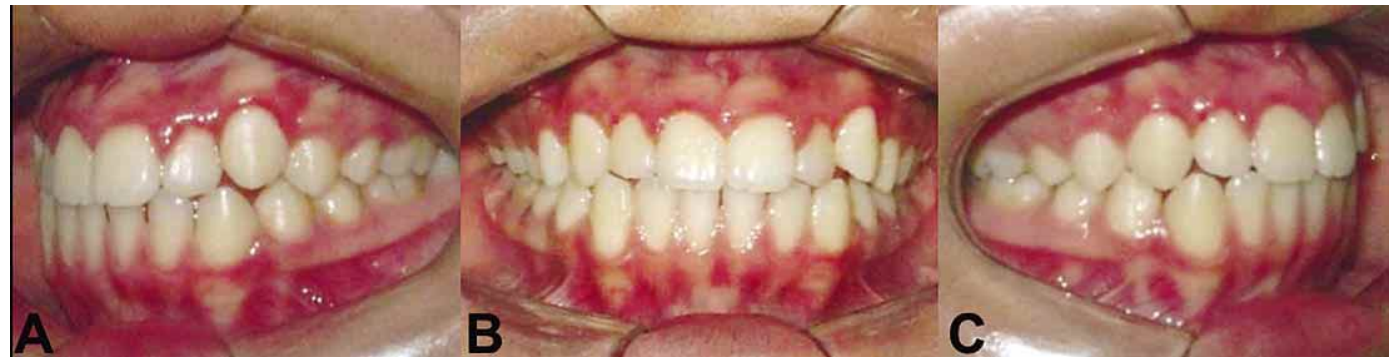

Fig. 4. A. Final lado izquierdo. B. Oclusión máxima final y C. Final lado derecho.

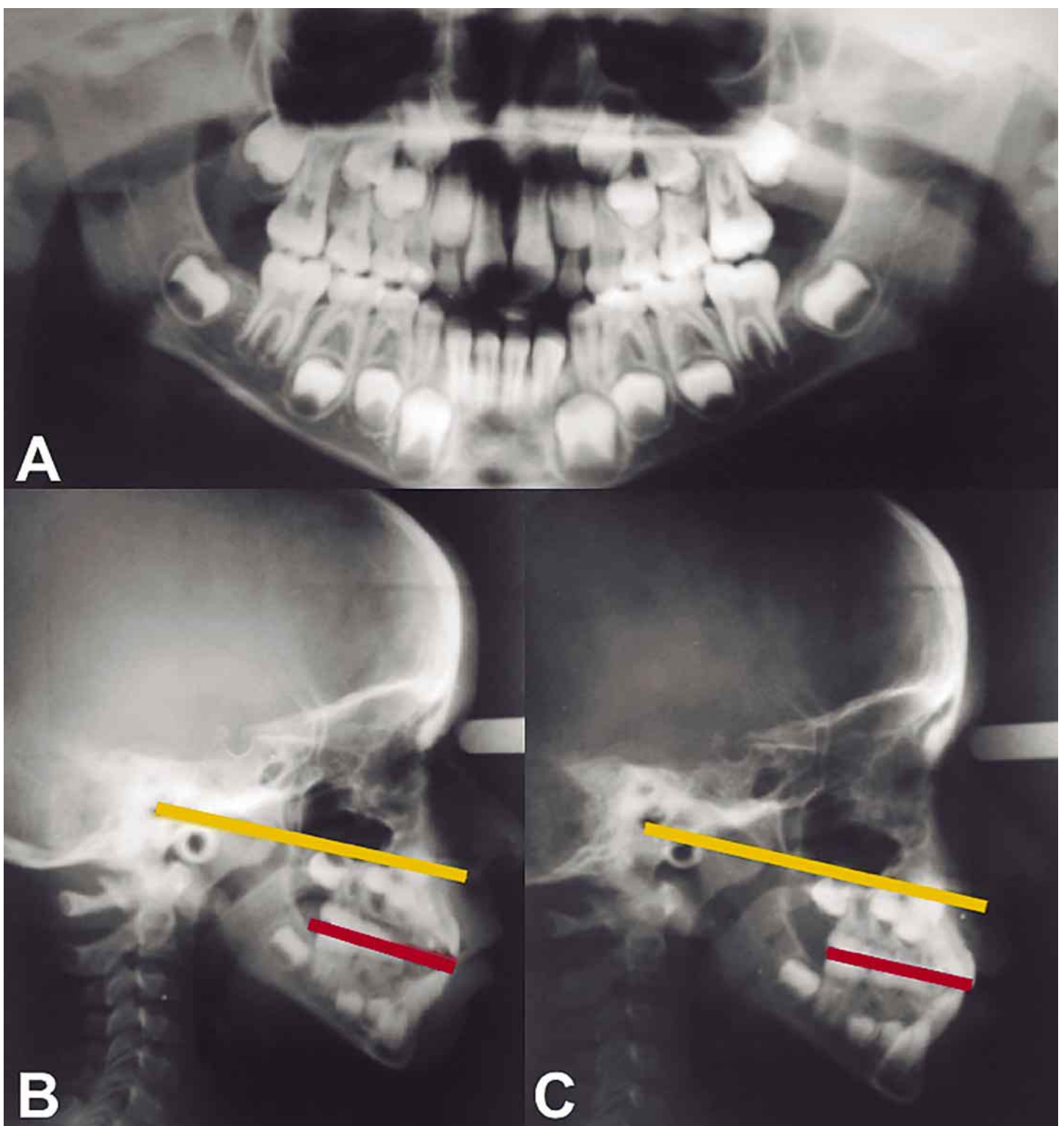

Fig. 5. A. Teleradiografía 2008. B. Ortopantomografía 2008 y C. Teleradiografía 2011.

En los estudios radiográficos iniciales (Fig. 5AB) se observa en la teleradiografía el paralelismo entre el plano oclusal (en rojo) y el plano de Camper (en amarillo), de pronóstico favorable. En la teleradiografía de control en el año 2011 (Fig. 5C), se observa la estabilidad en esta relación durante el tratamiento. 
La corrección de la mordida abierta se da por la elección de una terapéutica correcta y su estabilidad tiene su fundamento fisiológico en el reflejo linguomandibular (Guyton \& Hall, 2011). Al inicio el paciente presentaba pocos y pobres contactos dentarios que generaban contracciones musculares de baja intensidad, activando muy poco el reflejo, encargado de posicionar la lengua, que por lo tanto quedaba baja y adelantada, provocando la falta de contacto dentario anterior. Al aumentar la cantidad y calidad de los contactos dentarios a cada lado de la línea media, se au- menta la fuerza muscular al tiempo que se potencia el reflejo que acciona sobre la lengua, retruyéndola y permitiendo el cierre de la mordida abierta anterior.

\section{Segundo caso clínico}

Paciente de sexo masculino de 4 años de la clínica de prevención del IUCEDDU, presenta dentición caduca pura con una relación de clase I y un plano terminal recto. Mordida cubierta dentaria con una disfunción masticatoria asociada (6A-F).

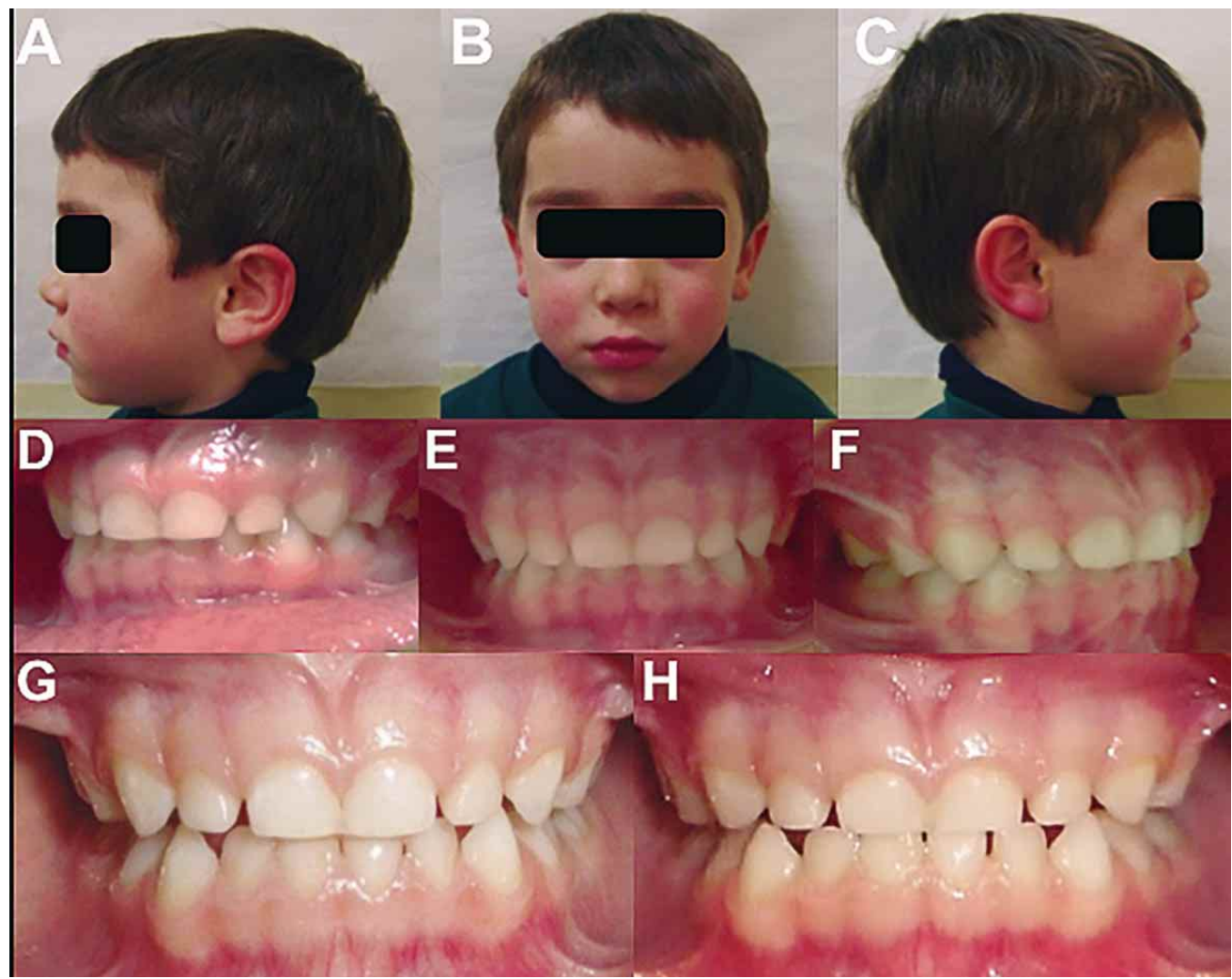

Fig. 6. A. Perfil izquierdo. B. Rostro inicial. C. Perfil derecho. D. Inicial lado izquierdo. E. Oclusión inicial. F. Inicial lado derecho. G. Inicio, confección de las pistas. H. Control al año. I. Ortopantomografía. J. Teleradiografía

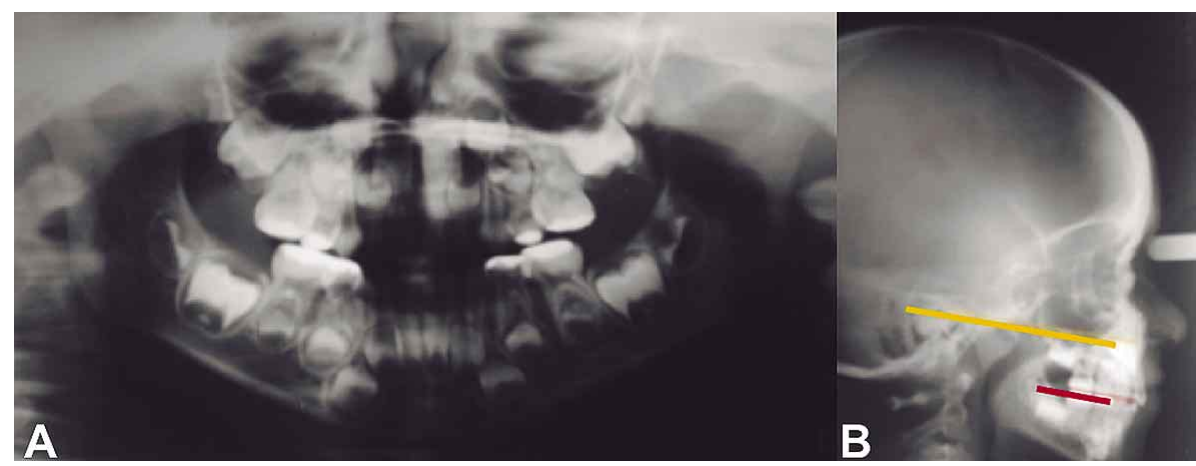

Fig. 7. Radiografía paorámica donde se observa la fórmula dentaria completa. B. En radiografía lateral se observa paralelismo entre el plano Oclusal (en rojo) y plano de Camper (en amarillo). 


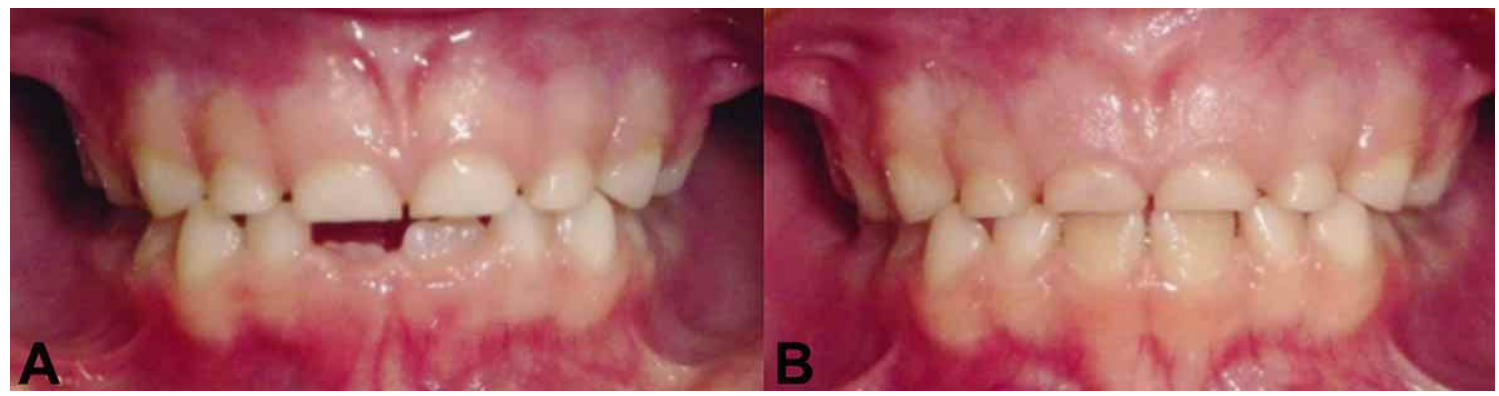

Fig. 8. A. Control a los 2 años. B. Control a los 3 años.

Plan de tratamiento: pistas directas Planas para aumentar la dimensión vertical, disminuyendo los ángulos funcionales masticatorios, transformando la masticación patológica en fisiológica para poder permitir que se realice la etapa de utilizacion y desgsate. Siempre complementado con ejercicios de Padovan (2007) para implementar lentamente los circuitos neurales reflejos de lateralidad mandibular durante el ciclo masticatorio (Fig. 6G-H).

Exámenes complementarios al año, nos muestran fórmula dentaria completa y paralelismo entre el plano Oclusal (en rojo) y plano de Camper (en amariIlo) (Fig. 7A-B). Esto se mantiene con el recambio dentario hacia dentición definitiva (Fig. 8A-B).

\section{CONCLUSIONES}

El equilibrado oclusal y las pistas directas Planas, basados en un correcto diagnóstico y respetando sus indicaciones clínicas, se presentan como un excelente recurso terapéutico para restablecer.la normalización funcional y promover condiciones para que el sistema estomatognático rescate su capacidad de autogerenciar el crecimiento y desarrollo que queda por delante. Esta terapéutica está indicada para correcciones dentarias y funcionales en niños en fase de dentición caduca y mixta temprana; tiene la capacidad de normalizar reflejos neuro-musculares patológicos así como la oclusión, posición mandibular y condilar dentro de las articulaciones témporomandibulares, logrando restablecer una función masticatoria fisiológica. Presenta la gran ventaja de mantenerse activos, independientemente de la colaboración del niño y de ser una técnica eficiente que requiere solamente la habilidad y conocimiento por parte del profesional que la realiza.

\section{AGRADECIMIENTOS}

A la Prof. Dra. W. Haller por su gran dedicación y grandeza al brindar por entero sus conocimientos a sus alumnos, al Dr. L. Carbajal, encargado del área de Investigación, por su apoyo constante en los trabajos científicos; al director académico del instituto, Dr. J.M. Delorenzi por su estímulo al crecimiento profesional.

CARBONE, I. L. Early malocclusion treatment without functional devices: Presentation of two clinical cases. Int. J. Odontostomat., 8(2):253-260, 2014.

ABSTRACT: This article outlines a model of Neuroocclusal rehabilitation (NOR) of Pedro Planas (1997) "it is fundamental to discover where, when and how we must act on the centers neural receptors that provide development of the stomatognathic system response for stimulating them physiologically provide us with a response of normal and balanced development". The NOR seeks to move from a pathological occlusal plane to a functional occlusal plane, changing the mandibular position, allowing growth and development eugnacico of the face. Clinical cases reported in this paper are relatively common in dental clinical practice, and their presentation seeks to emphasize the importance of early diagnosis of malocclusion with the therapeutic intervention; in order to prevent complications and simplify treatment, favoring a balanced morphofunctional growth and development in the patient, directly and positively reflected in their facial aesthetic. Two clinical cases are illustrated; one in deciduous dentition and the other in early mixed dentition, both from the IUCEDDU orthopedics and prevention clinic. Treatments with balanced occlusal and Planas direct tracks, making a longitudinal follow-up of the cases have been proposed. The results obtained in the treatments with this method, reaffirm our expectations and confirm that despite the use such simplified technique; excellent results are obtained that avoid or shorten subsequentfunctional treatment.

KEY WORDS: malocclusion, occlusal balance, planas direct tracks. 


\section{REFERENCIAS BIBLIOGRÁFICAS}

Barbosa Dos Santos, J. L. Curso teórico- clínico de pistas directas Planas. Montevideo, IUCEDDU, 2005.

Chibinski, A. C. R.; Czlusniak, G. D. \& Melo, M. D. Pistas directas planas: terapia ortopédica para corrección de mordida cruzada funcional. R. Clin. Ortodon. Dental Press, 4(3):64-72, 2005.

Deshayes, M.J. L'art de traiter avant 6 ans, 2006.

Guyton, C. G. \& Hall, J. E. Tratado de fisiología médica. 12a ed. Barcelona, Elsevier, 2011.

Padovan, B. El método Padovan como sistema terapéutico para las alteraciones funcionales orales y paraorales. Congreso Interno Ortopedia y Ortodoncia. Montevideo, IUCEDDU, 2007.

Planas, P. Reabilitação neuroclusal. $2^{\mathrm{a}}$ ed. Rio de Janeiro, Medsi, 1997.

Raymond, J. L. Finalité fonctionnelle et occlusale du traitement orthopédique de classe III. Rev. Orthop. Dento Faciale, 37(3):285-303, 2003.

Vesse, M. Traitement précoce de la classe III, les justifications théoriques et cliniques. Rev. Orthop. Dento. Faciale, 37(3):305-28, 2003.
Dirección para Correspondencia:

Dra. Lorena Carbone Irujo

Instituto Universitario Centro de Estudio y Diagnóstico de las Disgnacias del Uruguay (IUCEDDU)

Montevideo

URUGUAY

Email: lorenacarbone@adinet.com.uy

Recibido: 03-12-2013

Aceptado: 06-06-2014 